\title{
dialog-gesundheit - die Kraft der Frage für eine neue Gesundheitskompetenz
}

Michael Deppeler

Korrespondenz:

Dr. med. Michael Deppeler SaluToMed.AG Kirchlindachstrasse 7 CH-3052 Zollikofen Tel. 0319112060 Fax 0319116312 m.deppeler@hin.ch
Warum syt dir so truurig? Förchtet der das, wo no chönnt cho?

Aber dir syt doch versicheret gäge die mügleche Zuefäll Und wenn ds Alter de chunnt, heit dir de ä rächti Pension

Warum syt dir so truurig? Nei dir wüsset ke Grund

Vilicht, wenn der e Grund hättet, wäret der weniger truurig

Mänge, wenn ds Läben ihm wehtuet, bsinnt sech derdür wider dra

(Mani Matter)

Im Dezember 2009 feierten wir im Berner Bierhübeli 50 Jahre Berner Rock. Am Ende interpretierte Polo Hofer das letzte Lied von Mani Matter. Der immer noch provozierende Übervater der Mundart-Rockmusik setzte damit dem viel zu früh verstorbenen Troubadour ein weiteres Denkmal. Doch was haben diese beiden Musiker und Wortakrobaten mit dialoggesundheit und der Medizin zu tun? Sie erzählen Geschichten und sie stellen Fragen, die zum Nachdenken anregen.

Warum seid ihr so traurig? Sind wir Hausärzte auch traurig? Oder nur wütend? Und warum eigentlich? Haben wir andere Probleme und Sorgen als andere Menschen? Als unsere Patienten?

Brauchen wir in 10 Jahren noch Hausärzte? Mit dieser provozierenden und anregenden Frage wurden 5 Hausärzte im Jahr 2003 anlässlich der 20-Jahr-Feier der Berner Instanz für Hausarztmedizin konfrontiert. Welche Hausärzte brauchen wir in 10 Jahren? Und welche Grundversorgung, welche Medizin? In zwei moderierten Workshops, an denen auch der Hausarztmedizin nahestehende Kolleginnen und Kollegen teilnahmen, versuchten wir erste Antworten auf diese Fragen zu finden: Das Gesundheitswesen wird immer komplexer und kaum steuerbar, auf jeden Fall nicht «Von oben». Es ist einer der grössten Arbeitgeber und auf Gewinn angelegt, kaum jemand will wirklich sparen, aber: Wo bleiben die Patienten mit ihren Leiden? Auch im Workshop hatten wir den Patienten, um den sich eigentlich alles drehen sollte, immer wieder vergessen. Die schmerzhafte, aber letztendlich wertvolle Erfahrung des «vergessenen Patienten» wurde zum Ausgangspunkt von swissdoc, woraus sich in den letzten sechs Jahren dialog-gesundheit entwickelte (www. dialog-gesundheit.ch). Seither treffen sich Ärzte und Bevölkerung regelmässig zum Dialog über Gesundheitsfragen, die von den Patienten und aus ihrem Umfeld kommen, aus den Familien, Quartieren und Gemeinden. Dies fand erstmals statt im Jahr 2004 im
Forum dialog-gesundheit Zollikofen (ehem. swissdoc. forum). Seit 2006 trifft man sich im Forum dialoggesundheit Frutigland (im Jahr 2009 gab es eine Pause). Ein Forum findet alle zwei Monate statt, es dauert 90 Minuten, wird moderiert und zur Dokumentation protokolliert. Am Ende des Forums wird versucht, gemeinsam die Fragen und das Thema für das kommende Forum festzulegen. So entsteht eine Art bevölkerungsnaher «Qualitätszirkel zum Gesundheitswesen», ohne die Gefahr, dass seitens der Leitung über die Themenwahl zu stark gesteuert wird.

Ein Schlüsselerlebnis rund um dialog-gesundheit war der Vortrag von Prof. Syme (Universität Berkeley) an der 7. Schweizer Gesundheitsförderungskonferenz 2005 in Freiburg. Er erzählte seine persönliche Geschichte der Gesundheitsförderung und Prävention in Kalifornien, eine Geschichte des Scheiterns, des Suchens und des Findens neuer Wege. Auch er hatte sich um die bevölkerungsweite Krankheitslast (burden of disease) gekümmert, unzählige Präventionskampagnen gestartet und war vom mässigen Erfolg immer wieder enttäuscht worden. Nach einer Analyse der Misserfolge entschied er sich schliesslich, etwas Neues anzudenken: Er fragte die Bevölkerung nach ihren Bedürfnissen rund um die Gesundheit. Folgende - für uns Hausärzte nicht ganz überraschende - Antwort kam zutage: Wir haben ganz andere Problemen als ihr Gelehrten an den Universitäten denkt. (Ähnlich geht es wohl in der Politik.) Wir haben Sorgen mit der Erziehung, in der Partnerschaft, wir haben finanzielle Schwierigkeiten und ungelöste Konflikte am Arbeitsplatz, zumindest die von uns, die überhaupt noch eine Arbeit haben. Zuerst brauchen wir Unterstützung bei der Lösung dieser Alltagsprobleme, dann, aber erst dann, sind wir bereit, das Rauchen, Trinken oder Essen aufzugeben. Wir wissen, dass es uns nicht guttut.

So kam Kalifornien zu seinem ersten «Wellness guide». Nach der Evaluation kam das niederschmetternde Resultat. Er bewirkte ebenso wenig wie die anderen Präventionsprogramme. Wieder hatte Professor Syme Mut zum Feedback und fragte erneut: Was können wir bessermachen? Was ist unser Auftrag? Es zeigte sich, dass die Bevölkerung vom ersten Ratgeber völlig überfordert war - zu viel Text, zu kompliziert geschrieben. Sie wünschte sich kurze Texte, auch Bilder und klare Checklisten. Die zweite Ausgabe wurde zum Erfolg, vor allem bei der sozial schwächeren Bevölkerung. Die sozial bessergestellten, gebildeteren Personen suchen sich Lösungen ohnehin selbst, auch das erleben wir in der Sprechstunde. 
Parallel dazu ereignete sich im Jahr 2005 im Forum Zollikofen ganz Erstaunliches, als die Teilnehmer ihre drei Hauptbedürfnisse rund um die Gesundheit äusserten. Dies waren:

- Koordination der gesundheitsfördernden Angebote der Gemeinde und der Region. In der ersten dialog-gesundheit-Gegenwartskonferenz 2006 wurde diese Forderung erfüllt.

- Eine Ansprechperson in der Gemeinde, die über die Angebote im Gesundheitswesen niederschwellig Auskunft geben kann. Kurze Zeit später wurde eine 20\%-Stelle aus dem Stellenpool des Departements «Soziales», in dem auch die Gesundheit integriert ist, geschaffen.

- Bessere Information über Fragen rund um die Gesundheit. Der bisher bekannte «Chummerzhilf» mit den wenigen Notfalladressen war schon lange nicht mehr zeitgemäss. Seither arbeiten wir mit dialog-gesundheit am «Ratgeber für gesunde und kranke Tage», ein Projekt, das von der Gesundheitsförderung Schweiz als innovatives Projekt unterstützt wird.
Alltag, das ist das Leben. Zu diesem Leben gehört auch eine weitere Ebene, die oft ausgeklammert wird. Es ist die Ebene der Werte, oft auch eine spirituelle (nicht nur religiöse) Suche. Im vertrauten Rahmen eines Forums haben all diese Fragen und Dimensionen je länger je mehr Platz. Im Jahr 2009 wurde zum Beispiel auch über die Frage diskutiert: Was hat Versöhnung mit Gesundheit und Krankheit zu tun?

dialog-gesundheit will Gesundheitsförderung und Prävention direkt an der Basis, und diese zu Fragen und Sorgen, welche die Bevölkerung wirklich beschäftigen und belasten. Damit ergänzen wir all die «Topdown Lösungen», die in erster Linie in der Verhältnisprävention (z.B. Rauchverbot, Werbeverbote usw.) eine Wirkung erzielen, leider aber nur sehr wenig in der Verhaltensprävention - hier ist ein anderer Weg, ein neues Denken gefragt. In einem länger dauernden Prozess wird der Patient, die Bevölkerung, zu Eigenund Mitverantwortung eingeladen und motiviert: im Forum - im Dialog, mit der Zeit auch in anderen Bereichen von Public health und zuletzt ganz «organisch gewachsen» auch in der Sprechstunde. Wir alle haben

\section{Probleme kann man niemals mit der gleichen Denkweise lösen, durch die sie entstanden sind (Albert Einstein)}

Diese sehr eindrücklichen und vor allem basisnahen Schritte in einem moderierten und protokollierten Dialog führten dazu, dass nicht nur an einer individuellen, sondern auch kollektiven Gesundheitskompetenz gearbeitet wird. Diese lehnen sich an die heute vieldiskutierten Gesundheitsdeterminanten an. Sie spiegeln eindrücklich wider, was theoretisch in den letzten Jahrzehnten erforscht und erkannt worden ist: Die Gesundheit und die Krankheiten dürfen nicht mehr auf einfache monokausale Ursache-Wirkungsprinzipien reduziert werden. Es handelt sich immer um komplexe biopsychosoziale Prozesse, die systemische Ebene muss mitberücksichtigt werden. Das ist der

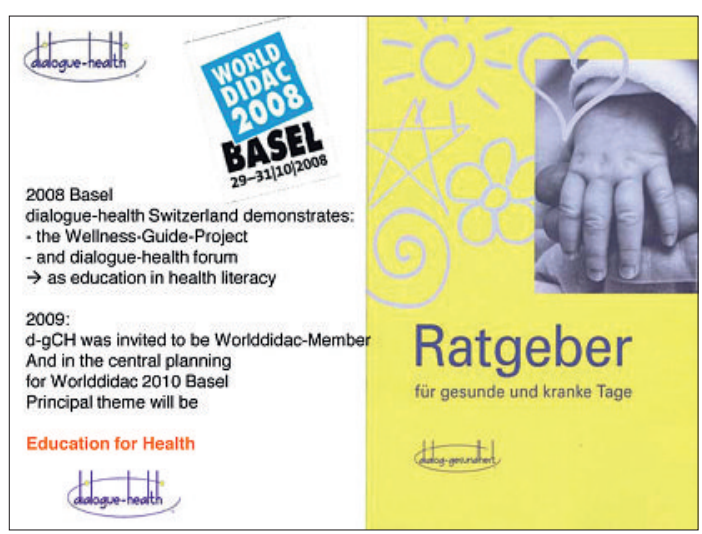

Der Ratgeber mit Informationen zu wichtigen Fragen rund um die Gesundheit entstand auf Anregung der Bevölkerung. so auch die Möglichkeit - die Freiheit - neue Lösungen und Ressourcen zu entdecken in einer komplexen Welt, die immer mehr durch neue Gesetze, Verordnungen und Weisungen «fremdbestimmt» wird. So werden auch dringend notwendige Public-healthsowie sozial-gesundheits- und gesellschaftspolitische Aspekte gestärkt, insbesondere auch neben den gewinn- und marktwirtschaftlich orientierten Strategien anderer «Players». Aus diesem Grund ist es dialoggesundheit auch wichtig, wirklich unabhängig zu bleiben. Dazu gehört auch der Aufbau einer eigenen «Wissen schaffenden» Plattform, die den gesamten Prozess, aber auch Teilaspekte davon, evaluieren soll. Im Grunde genommen ist dialog-gesundheit ein grosser systemischer Kommunikations- und Lösungsprozess, der - so denken wir - letztendlich sicher zweckmässig, wirksam und, wer weiss, sogar wirtschaftlich sein wird. Schon allein deswegen, weil wir bisher alle nach den Richtlinien der Freiwilligenarbeit gearbeitet haben, doch wird das immer so bleiben? Ist unsere Arbeit auch so wertvoll genug - und das für alle Mitwirkenden?

Eine sehr wichtige Erkenntnis in den Jahren 2008 und 2009 war der Wunsch der Bevölkerung nach einer besseren Verknüpfung von Gesundheit und Bildung. Dies schon in der Schule, aber dann vor allem ein Leben lang durch Erwachsenenbildung, gerade im quartären Bereich wie z.B. auch in Form von dialoggesundheit mit seinen Foren. Auch in der Bildung 
sind neue Wege gefragt, sie ähneln erstaunlich (?) den neuen Ansätzen im Gesundheitswesen. Weg vom «partriarchalischen Lehrer-Schüler-Verhältnis» hin zum gemeinsamen Entwickeln neuer Kompetenzen. Aus der Resilienzforschung ist dabei der Begriff der stärkenden Lerndialoge (Sustained shared thinking) bekannt: Es geht um ein gemeinsames, nachhaltiges Denken zwischen Erzieher und Kind, um einen bewusst dialogischen Denkprozess, in dem offene Fragen (!), die zum Nachdenken, zum Problemlösen oder zum Dialog anregen, verwendet werden sollten. Offenbar sind 95\% der Fragen in den Schulen geschlossen. Sind wir im Gesundheitswesen wirklich sehr viel weiter? Im stärkenden Lerndialog wird das Bewusstsein für die Interessen und die Perspektiven gefördert, ein Dialog auf Augenhöhe, ein aktives Zuhören und eine neue Achtsamkeit, damit wirklich Brücken gebaut werden können. Jeder erlebt und versteht sich selbst als Lernender. Der andere ist Spezialist seines Lebens und seiner Geschichte. Gemeinsam können wir viel voneinander lernen.

Der Wert des «Geschichtenerzählens» (narrative Medizin) ist ja schon lange und auf verschiedensten Ebenen erkannt worden, auch in der Hausarztmedizin. Es braucht kein besonderes Material, z. B. auch keine PowerPoint-Präsentationen und keinen Beamer. Im Forum sitzen wir im Kreis, für wichtige, wegweisende Erkenntnisse (besonders auch zur Unterstützung des Protokolls) dient ein Flipchart. Die Kraft des Erzählens und des Zuhörens genügt. Die Teilnehmer erwerben durch das wiederholte Üben vor anderen Menschen auch die Fähigkeit, ihre Sorgen und Ängste, aber auch ihre Bedürfnisse, ihre Geschichten besser auszudrücken, was auch der Sprechstunde zugute kommt. Das Erzählen geschieht auf drei Ebenen: Eine Mitwirkende erzählt ihre Geschichte und wie sie ihre Lebensaufgabe löst. Der Moderator erzählt Geschichten, die unter anderem Lösungsideen beinhalten. Die Beziehung zwischen den Menschen und innerhalb der Gruppe gestaltet ihre eigene Geschichte. Das gemeinsame Teilen und Mitteilen von Leiden führt zu neuen, alternativen Erzählungen, die den Handlungsspielraum der Beteiligten, der Gruppe, erweitern und so helfen können, ihre Probleme zu lösen und letztendlich gesünder zu leben.

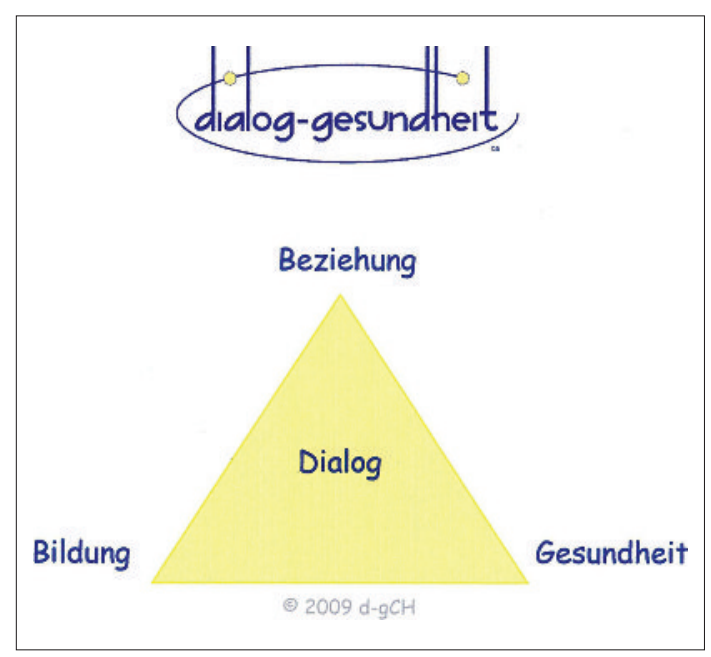

Damit wird dialog-gesundheit immer auch salutogenetisch. Gerade dieser Ansatz kann entscheidend sein, wenn es darum geht, Gesundheitsförderung und Prävention wirklich ins Blickfeld von Politik und Bevölkerung zu bringen. Dabei geht es um drei Ebenen: - Verstehen: Jemand muss etwas verstehen können. Das ist abhängig von Sprache und Kultur. Hier braucht es niederschwellige Möglichkeiten von der Schule bis ins Alter. Information und Kommunikation sind zentral.

- Handeln: Wo sind die Ressourcen, also Stärkung der Copingstrategien und des Selbstmanagements, Unterstützen des persönlichen Entwicklungs- und Reifungsprozesses, aber auch das Teilhaben an «externen Ressourcen», dazu gehört unter anderem auch die optimierte Koordination der «Helfer» (integrierte Versorgung).

- Sinn: Was tut mir gut, was nicht? Was macht Sinn für mich und mein Umfeld, für was lebe ich, wofür will ich gesund bleiben oder werden?

Warum syt dir so truurig? Wohl, me gseht nech's doch a. Söttet emal öiji Gsichter gseh, wenn dr sitzet im Büro. Söttet emal öiji Gsichter gseh, wenn der fahret im Tram. Warum syt dir so truurig? S'geit doch so wi der's weit. Frou u Chind sy doch zwäg, im Pruef geit's geng e chly vorwärts,

s'längt doch ou hie und da scho für nes chlys Drübery. (Mani Matter) 